\title{
The tumour-suppressive miR-29a/b / cluster is regulated by CEBPA and blocked in human AML
}

\author{
M Eyholzer ${ }^{1,2}$, S Schmid ${ }^{2}$, L Wilkens ${ }^{3}$, BU Mueller ${ }^{1,4}$ and T Pabst ${ }^{*, 2}$ \\ 'Department of Clinical Research, University of Bern, Bern, Switzerland; '2Department of Medical Oncology, Inselspital, Bern University Hospital, University \\ of Bern, Bern, Switzerland; ${ }^{3}$ Department of Pathology, Hospitals of the Region Hannover, Hannover, Germany; ${ }^{4}$ Department of Internal Medicine, \\ Bern University Hospital, University of Bem, Bern, Switzerland
}

BACKGROUND: CCAAT/enhancer-binding protein- $\alpha$ (CEBPA) is crucial for normal granulopoiesis and is frequently disrupted in acute myeloid leukaemia (AML). Increasing evidence suggests that CEBPA exerts its effects, in parts, by regulating specific microRNAs (miRNAs), as previously shown for miR-223. The aim of this study was to investigate the genome-wide pattern of miRNAs regulated by CEBPA in myeloid cells.

METHODS: In Kasumi-I cells, conditionally expressing CEBPA, we assessed the expression of 470 human miRNAs by microarray analysis. We further investigated the microarray results by qRT-PCR, luciferase reporter assays, and chromatin immunoprecipitation assays.

RESULTS: In all, 18 miRNAs were more than two-fold suppressed or induced after CEBPA restoration. Among these 18 miRNAs, we focused on CEBPA-mediated regulation of the tumour-suppressive miR-29b. We observed that miR-29b is suppressed in AML patients with impaired CEBPA function or loss of chromosome $7 \mathrm{q}$. We found that CEBPA selectively regulates miR-29b expression on its miR-29a/b/ locus on chromosome 7q32.3, whereas miR-29b2/c on chromosome lq32.2 is not affected.

CONCLUSION: This study reports the activation of the tumour-suppressive miR-29b by the haematopoietic key transcription factor CEBPA. Our data provide a rationale for miR-29b suppression in AML patients with loss of chromosome 7q or CEBPA deficiency. British Journal of Cancer (2010) 103, 275-284. doi:10.1038/sj.bjc.660575I www.bjcancer.com

(c) 2010 Cancer Research UK

Keywords: AML; CEBPA; miR-29a/b/c family; transcriptional regulation

Haematopoiesis is a highly orchestrated interaction of lineagespecific transcription factors driving pluripotent precursor cells to differentiate towards mature blood cells (Rosenbauer and Tenen, 2007). Increasing evidence suggests that this differentiation along the various haematopoietic lineages is, in part, also regulated by microRNAs (miRNAs) (Lawrie, 2007; Garzon and Croce, 2008; Pelosi et al, 2009). miRNAs are small, non-coding RNAs, which silence target genes by base-pairing to untranslated mRNA regions. Thereby, miRNAs adjust expression of specific transcription factors in a post-transcriptional manner (Shivdasani, 2006; Ambros and Chen, 2007). Deregulation of either haematopoietic transcription factors or miRNAs is a common event in the molecular pathogenesis of human leukaemias (Tenen, 2003; Kluiver et al, 2006; Rosenbauer and Tenen, 2007; Fabbri et al, 2008).

One of the key transcription factors for normal haematopoiesis is the CCAAT/enhancer-binding protein- $\alpha$ (CEBPA). It has been shown to be crucial for myeloid differentiation towards mature granulocytes (Zhang et al, 1997; Radomska et al, 1998). In human acute myeloid leukaemia (AML), CEBPA function is frequently disrupted (Pabst and Mueller, 2007). Approximately $10 \%$ of AML patients show dominant-negative mutations in the CEBPA coding region (Pabst et al, 2001b). In addition, CEBPA expression is suppressed by the leukaemogenic fusion proteins AML1-ETO, AML1-MDS1-EVI1, or CBFB-SMMHC in AML patients bearing the

*Correspondence: Dr T Pabst; E-mail: thomas.pabst@insel.ch

Received 9 March 20I0; revised 26 May 20 I0; accepted 27 May 2010 chromosomal rearrangements $t(8 ; 21), t(3 ; 21)$ or inv(16) respectively (Pabst et al, 2001a; Helbling et al, 2004, 2005).

During normal haematopoiesis, various CEBPA downstream effectors have been described (Tenen, 2003; Mueller and Pabst, 2006), including so far at least one miRNA (miR-223) (Fazi et al, 2005; Fukao et al, 2007; Eyholzer et al, 2009). The activation of miR-223 by CEBPA can trigger neutrophil differentiation and is necessary for maintaining proper function of mature neutrophils (Fazi et al, 2005, 2007; Johnnidis et al, 2008). On the basis of these reports and the prominent role of CEBPA for normal myelopoiesis, we assessed in this study the genome-wide regulation of miRNAs by CEBPA in myeloid leukaemic cells. We screened 470 human miRNAs for their expression levels in CEBPA-deficient leukaemic Kasumi-1 cells using a conditional CEBPA expression system. We identified 18 miRNAs whose expression levels changed more than two-fold after CEBPA induction. Among them, we identified the tumoursuppressive $m i R-29 a / b 1$ cluster to be a direct target of CEBPA.

\section{PATIENTS AND METHODS}

\section{Patients, controls and cell lines}

Bone marrow samples from 66 consecutive AML patients collected at diagnosis before treatment were used, comprising all FAB subtypes. Leukaemic cells were collected using Ficoll gradient (Lymphoprep; Axis-Shield PoC AS, Oslo, Norway). miRNA was extracted using the miRNeasy Mini kit no. 217004 (Qiagen AG, 
Hombrechtikon, Switzerland). Mature monocytes or granulocytes from six healthy volunteers were isolated from peripheral blood using the EasySep selection kits nos. 18088-CD14 and 18682CD66b (RoboSep; StemCell Technologies, Vancouver, Canada). $\mathrm{CD} 34+$ myeloid stem cells from three patients were enriched using the CliniMacs CD34 Complete kit no. 177-01 (Miltenyi Biotec, Auburn, CA, USA). Informed consent from patients and volunteers was obtained according to the Declaration of Helsinki Principles. Clinical characteristics are summarised in Supplementary Table S1 (Supplementary Material).

Leukaemic Kasumi-1 cells stably transfected with an inducible CEBPA-oestrogen receptor (ER) fusion construct (CEBPA-ER) (Pabst et al, 2001a) were cultured in phenol red-free RPMI 1640 supplemented with $10 \%$ foetal calf serum (FCS). The CEBPA-ER fusion protein was activated using $1 \mu \mathrm{M} \beta$-oestradiol. All reagents were from Sigma-Aldrich (Buchs, Switzerland).

Leukaemic U937 cells stably transfected with the tetracyclineinducible (tet-off) oncogenic $t(8 ; 21)$ fusion protein AML1-ETO (Pabst et al, 2001a) were cultured in RPMI 1640 supplemented with $10 \%$ FCS and $0.75 \mu \mathrm{g} \mathrm{ml}^{-1}$ tetracycline. To induce AML1-ETO expression the cells were extensively washed with PBS and cultured in RPMI 1640, supplemented with 10\% tetracycline-free FCS (PAA Laboratories $\mathrm{GmbH}$, Pasching, Austria).

Leukaemic HL60, K562, Kasumi-1 and U937 cells, and H1299 lung cancer cells (ATCC, Manassas VA, USA) were cultured in RPMI 1640 with $10 \%$ FCS. The cell lines were characterised by molecular diagnostics and cytogenetics, and cell morphology was monitored by microscopy according to ATCC guidelines (http://www.atcc.org $>$ cultures and products $>$ technical support $>$ technical literature $>$ technical bulletin no. 8). No abnormalities in cell morphology were observed in these cell lines, both at low and high densities of cultures during the course of these experiments. Repetitive mycoplasma screening remained negative in these cell lines (PCR mycoplasma test kit Promokine no. PK-CA91-1048; PromoCell GmbH, Heidelberg, Germany).

\section{miRNA microarray}

Parental Kasumi-1 and Kasumi-1 cells with the inducible CEBPA-ER construct were collected before and $72 \mathrm{~h}$ after $\beta$-oestradiol treatment. miRNA was extracted using the miRNeasy mini kit no. 217004 (Qiagen AG), and miRNA quality was determined using Agilent 2100 Bioanalyzer (Agilent Technologies, Basel, Switzerland).

To assess miRNA expression profiles, we used the human miRNA microarray kit no. G4470A, detecting 470 human and 64 viral miRNAs based on the Sanger database version 9.1 (http://www.mirbase.org). Scanning and image analysis were carried out using the Agilent DNA microarray scanner (no. G2565BA; XDR 5/100, single pass, green). Feature Extraction software (version 9.5; Agilent Technologies) was used for data extraction from raw microarray image files using the miRNA_v1_95_May07 FE protocol (grid 016436_D_20070426). Data analysis was carried out using GeneSpring GX 9.0 (Agilent Technologies) expression analysis software and expression values were corrected for oestrogen effects. The cut-off for miRNA candidates was set at more than two-fold changes in expression (suppression or induction) after CEBPA restoration, and such changes had to be observed in two independant experiments. The microarray kit, equipment and software used for these arrays were from Agilent Technologies.

\section{Luciferase reporter assay}

The human DNA sequence comprising -682 to +296 bp upstream of the primary $m i R-29 a / b 1$ transcription start site (GenBank accession number EU154353) was cloned into the pGL3b luciferase vector using $K p n I$ and NheI restriction sites. This construct was co-transfected with a human CEBPA expression plasmid (pcDNA3) in H1299 cells using Lipofectamine 2000 (Invitrogen, Basel, Switzerland). Luminescence was detected using the Dual-Luciferase Reporter Assay (Promega, Dübendorf, Switzerland). Primer sequences are indicated in Supplementary Table S2 (Supplementary Material).

\section{Quantitative RT-PCR}

$m i R-29 b$ expression in samples from AML patients and healthy volunteers was assessed using the miScript SYBR Green PCR kit no. 218073 and primer assay hs-miR-29b no. MS_6566 (Qiagen AG). Expression values were normalised to the geometric mean (Peltier and Latham, 2008) of $m i R-93$ and $m i R-191$ expression (nos. MS_3346 and MS_3682 respectively; Qiagen AG). To distinguish between $m i R-29 a$ and $m i R-29 c$ expression, we used TaqMan microRNA assays no. 001212 (29a) and no. 000578 (29c) and TaqMan universal PCR master mix No AmpErase UNG no. 4324018 (Applied Biosystems, Rotkreuz, Switzerland). Primer sequences for pri-miR-29a/b/c detection using QuantiTect SYBR Green PCR kit no. 204143 (Qiagen AG) are indicated in Supplementary Table S2 (Supplementary Material). Expression values of $m i R-29 a / b / c$ and their primary transcripts in cell line experiments were normalised to $m i R-93$ expression, as $m i R-93$ showed robust and stable expression during the time courses in this study. All qRT-PCR reactions were carried out on 7900HT Fast Real-Time PCR system (Applied Biosystems).

\section{Chromatin immunoprecipitation assay}

Chromatin immunoprecipitation assays were performed using the ChIP-IT Express Enzymatic kit no. 53009 (Active Motif, Rixensart, Belgium). Immunoprecipitation of sheared chromatin of parental U937 as well as of Kasumi-1-CEBPA-ER cells collected $72 \mathrm{~h}$ after $\beta$-oestradiol treatment was performed using antibodies against CEBPA (polyclonal rabbit IgG, sc-61X), polymerase II (sc-900X), and rabbit IgG (sc-2027; all from Santa Cruz, Heidelberg, Germany). Sequences of the PCR primers to detect CEBPA binding to the pri-miR-29a/b1 promoter or to the pre-miR-223 regulatory element as positive control (Fazi et al, 2005) are described in Supplementary Table S2 (Supplementary Material).

\section{Western blot analysis}

Protein detection was carried out from whole-cell lysates using antibodies against CEBPA no. 39306 (1:500; Active Motif) and DNMT3B sc-10236 (1:500; Santa Cruz). For loading control, $\beta$-actin antibody MAB1501 (1:105; Chemicon/Milipore, Zug, Switzerland) was used. Horseradish-peroxidase-linked secondary antibodies (1:5000 each) were: anti-mouse no. NA931V, anti-rabbit no. NA934V (Amersham, GE Healthcare Bio-sciences, Uppsala, Sweden), and anti-goat sc-2020 (Santa Cruz).

\section{URL and statistical analysis}

Conservation studies of the pri-miR-29a/b1 (GenBank accession number EU154353) and pri-miR29b2/c (EU154351 and EU154352) loci were carried out using http://www.genome.ucsc.edu/ (assembly March 2006). Promoter analysis for putative CEBP binding sites were performed using Genomatix MatInspector software, release 7.7(3) (Genomatix Software GmbH, Munich, Germany). Differences in promoter activities and miR-29 expression levels were analysed by $t$-test, with $P<0.05$ defining significance using GraphPad Prism software version 4.0 (GraphPad Software Inc., La Jolla, CA, USA).

\section{RESULTS}

Genome-wide changes in miRNA expression after CEBPA restoration in human AML

To identify miRNAs regulated by the myeloid key transcription factor CEBPA in the haematopoietic system, we carried out miRNA 
microarrays assessing 470 human miRNAs. We used leukaemic Kasumi-1 cells lacking detectable amounts of endogenous CEBPA, however, containing an inducible CEBPA-ER fusion construct (Pabst et al, 2001a). We treated these cells with $\beta$-oestradiol for $72 \mathrm{~h}$ to restore the CEBPA function, and analysed the changes in miRNA expression. We found that the expression of 18 miRNAs changed more than two-fold after restoring CEBPA function compared with untreated Kasumi-1-CEBPA-ER cells and after exclusion of effects because of oestrogen treatment (Table 1). Of the 18 miRNAs, 8 (44\%) were suppressed (Table 1A: miR-98, $m i R-181 b, m i R-197, m i R-210, m i R-342, m i R-432, m i R-550$, and $m i R-776)$, whereas 10 miRNAs (56\%) were induced (Table 1B: miR-29b, miR-223, miR-370, miR-496, miR-572, miR-575 miR-630, miR-638, miR-663, and miR-765; Supplementary Table S3). $m i R-223$, a previously identified target of CEBPA (Fazi et al, 2005; Fukao et al, 2007; Eyholzer et al, 2009), was confirmed and used as a positive control for the array experiments, with a twofold induction after restoring CEBPA in our cell line model.

With a focus on haematopoiesis, differentiation, and/or carcinogenesis, we summarised the rapidly increasing literature available for the 18 identified miRNAs in Table 1 (for references see also Supplementary Table S3). Most of these reports describe expression patterns in various types of normal tissues and cancer, whereas reports on the regulation of specific miRNAs are rare.

In this study, we focused on CEBPA-regulated miRNAs with tumour-suppressive functions in haematopoiesis, and $m i R-29 b$ represented the most prominent candidate. $m i R-29 b$ belongs to a miRNA family comprising three members ( $m i R-29 a,-29 b$, and $-29 c$ ), which have been reported to be suppressed in various cancer types (Fabbri et al, 2007; Mott et al, 2007; Wang et al, 2008), including leukaemias (Li et al, 2008; Stamatopoulos et al, 2009; Garzon et al, 2009b). Furthermore, they were shown to induce differentiation (Wang et al, 2008; Li et al, 2009; Garzon et al, 2009b) and apoptosis (Park et al, 2009), and inhibit epigenetic silencing due to de novo methylation (Fabbri et al, 2007; Garzon et al, 2009b).

\section{CEBPA mediates $m i R-29 b$ expression in AML}

We aimed to verify the results of the miRNA microarray by qRT-PCR. We observed that $m i R-29 b$ was, indeed, induced twofold after CEBPA restoration in the Kasumi-1-CEBPA-ER cell line system (Figure 1A). We then investigated the effect of CEBPA knock down on miR-29b expression. We used parental U937 leukaemic cells, expressing high levels of endogenous CEBPA as well as a tet-off system conditionally expressing the oncogenic $\mathrm{t}(8 ; 21)$ fusion protein AML1-ETO (Pabst et al, 2001a). Induction of AML1-ETO in these cells efficiently blocked CEBPA protein expression (Figure 1B, left), which led to suppressed miR-29b expression (Figure 1B, right).

Interestingly, CEBPA-associated expression of $m i R-29 b$ was further observed across a variety of leukaemic cell lines: the expression of CEBPA protein and $m i R-29 b$ in HL60, K562, Kasumi-1, and U937 cells inversely correlated to the protein expression of the previously identified $m i R-29 b$ target gene DNA methyltransferase 3B (DNMT3B, Figure 1C; Fabbri et al, 2007; Garzon et al, 2009b). As DNMT3B is mediating de novo DNA methylation and thus epigenetically inactivates tumour suppressor genes in cancer (Robertson et al, 1999; Rhee et al, 2002; Lin et al, 2007), these observations connect blocked differentiation through CEBPA suppression with deregulated methylation because of the suppressed $m i R-29 b$ activity.

\section{miR-29b expression is suppressed in AML patients with impaired CEBPA function or with monosomy 7 or $\operatorname{del}(7 q)$}

To evaluate the importance of CEBPA-mediated $m i R-29 b$ induction in vivo, we analysed $m i R-29 b$ expression in diagnostic samples of 66 AML patients, three samples of enriched CD34 + myeloid stem cells, and in samples of mature granulocytes and monocytes from 6 healthy volunteers (Figure 2).

We observed that the mean expression of $m i R-29 b$ was suppressed in the entire cohort of AML patients compared with normal granulocytes $(P=0.043)$. In our cohort, we then separately analysed the patients with suppressed CEBPA function. This group comprised the AML patients with CEBPA mutations, with $\mathrm{t}(8 ; 21)$ or with inv(16) (Pabst et al, 2001a,b; Helbling et al, 2005). We found that these AML patients had suppressed miR-29b compared with mature granulocytes $(P=0.0001$ for CEBPA mutated, $P<0.0001$ for $\mathrm{t}(8 ; 21)$ and inv(16)). Remarkably, the low $m i R-29 b$ levels were comparable with $m i R-29 b$ expression in CD34+ precursor cells, which hardly express detectable amounts of CEBPA (Radomska et al, 1998).

In addition, we confirmed previous observations (Garzon et al, 2009 a) that $m i R-29 b$ is suppressed in patients with monosomy 7 or $\operatorname{del}(7 \mathrm{q})(P=0.012)$. On combining AML patients with alterations of chromosome 7q or CEBPA $(n=21)$, we observed low $m i R-29 b$ expression compared with the remaining 45 patients of our cohort, with other or no detectable genomic alterations $(P=0.0002)$. We thus confirmed in our cohort of 66 AML patients that miR-29b expression is associated with CEBPA levels and therefore suppressed in patients with disrupted CEBPA function.

\section{Only $m i R-29 a / b 1$ is induced after restoring CEBPA in human AML cells}

$m i R-29 b$ belongs to the miR-29 family that is encoded in two clusters on two chromosomes (Figure $3 \mathrm{~A}$ ): $m i R-29 a$ as well as $m i R-29 b$ on chromosome $7 \mathrm{q} 32.3$, and $m i R-29 c$ as well as, again, $m i R-29 b$ on chromosome 1q32.2. Mature $m i R-29 b$ is therefore encoded by two distinct precursor stem sequences (pre-miRNA) on both chromosomes, a pre-miR-29b1 and pre-miR-29b2 stem. Although the sequences of the two pre-miR-29b stems are differing, mature $m i R-29 b$ resulting from these two stem structures is identical.

Consequently, we first investigated the transcriptional effects of CEBPA on the two miR-29 loci to define the individual contribution of each locus to $m i R-29 b$ expression. Our microarray data indicated a roughly two-fold induction of $m i R-29 a$ expression after restoring CEBPA in Kasumi-1 cells, whereas $m i R-29 c$ tended to be suppressed (-1.3-fold). By qRT-PCR, we confirmed that $m i R-29 a$ was induced two-fold $72 \mathrm{~h}$ after CEBPA restoration, similarly to $m i R-29 b$. In contrast, the expression of $m i R-29 c$ was not affected (Figure 3B). This suggests that $m i R-29 a$ and $m i R-29 b$, but not $m i R-29 c$, are regulated by CEBPA.

Previous reports (Chang et al, 2008; Wang et al, 2008) indicated that mature miR-29 family members encoded on the same chromosome are processed from a common primary transcript (pri-miRNA, Figure 3C). We thus designed a series of primer pairs dispersed over the pri-miR-29a/b1 and pri-miR-29b2/c sequences. Again, we observed a two-fold induction of pri-miR-29a/b1 (Figure 3D, left), whereas the expression of the $m i R-29 b 2 / c$ primary transcript remained stable (Figure $3 \mathrm{D}$, right). This is consistent with the above-mentioned observation of suppressed $m i R-29 b$ in AML patients with aberrant chromosome 7q. We thus concluded that CEBPA activates the expression of the $m i R-29 a / b 1$ cluster on chromosome 7, whereas it does not affect $m i R-29 b 2 / c$ on chromosome 1 in myeloid leukaemic cells.

\section{CEBPA specifically activates the pri-miR-29a/b1 promoter}

As previously shown by RACE experiments (Chang et al, 2008), the primary $m i R-29 a / b 1$ transcript starts $35.7 \mathrm{~kb}$ upstream of the pre-miR-29b1 stem structure, and the highly conserved promoter region just upstream of this transcription start is responsible for regulation of $m i R-29 a / b 1$ expression (Figure 3C). 


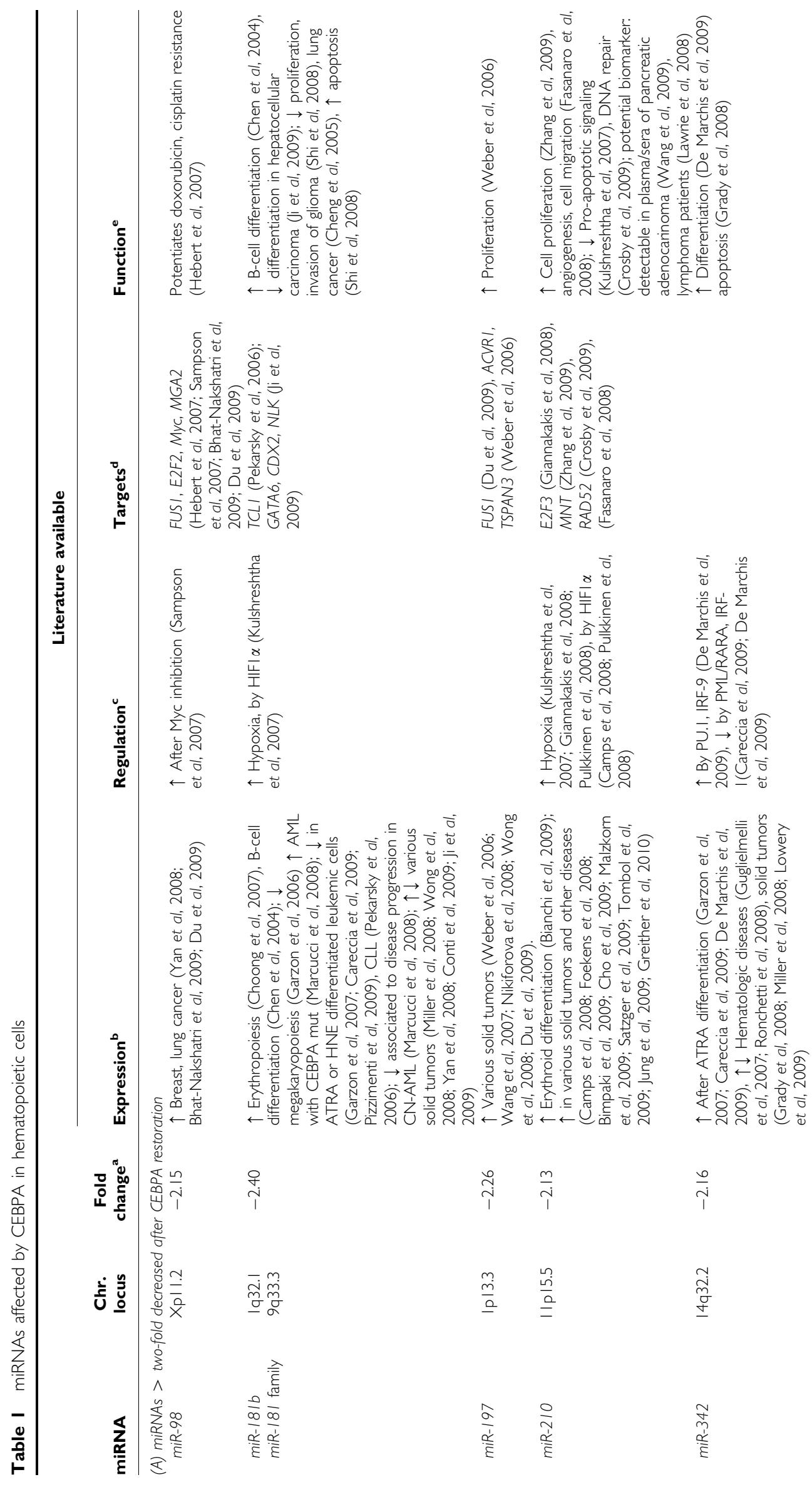

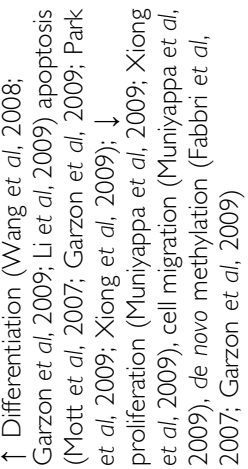

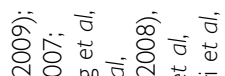

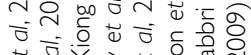

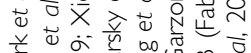

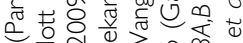

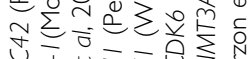

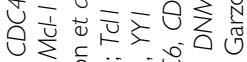

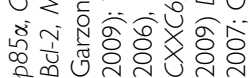

苞之

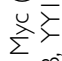

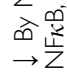

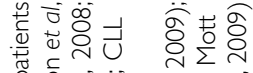

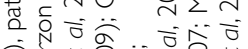

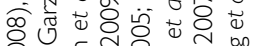
V.

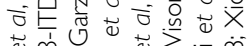
च.

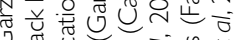

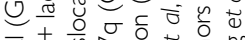

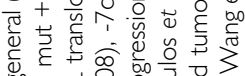

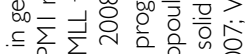

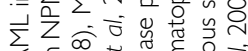

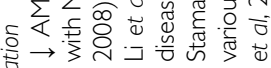

îi i

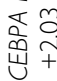
產

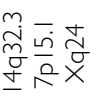

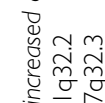

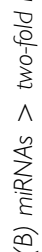

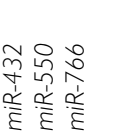




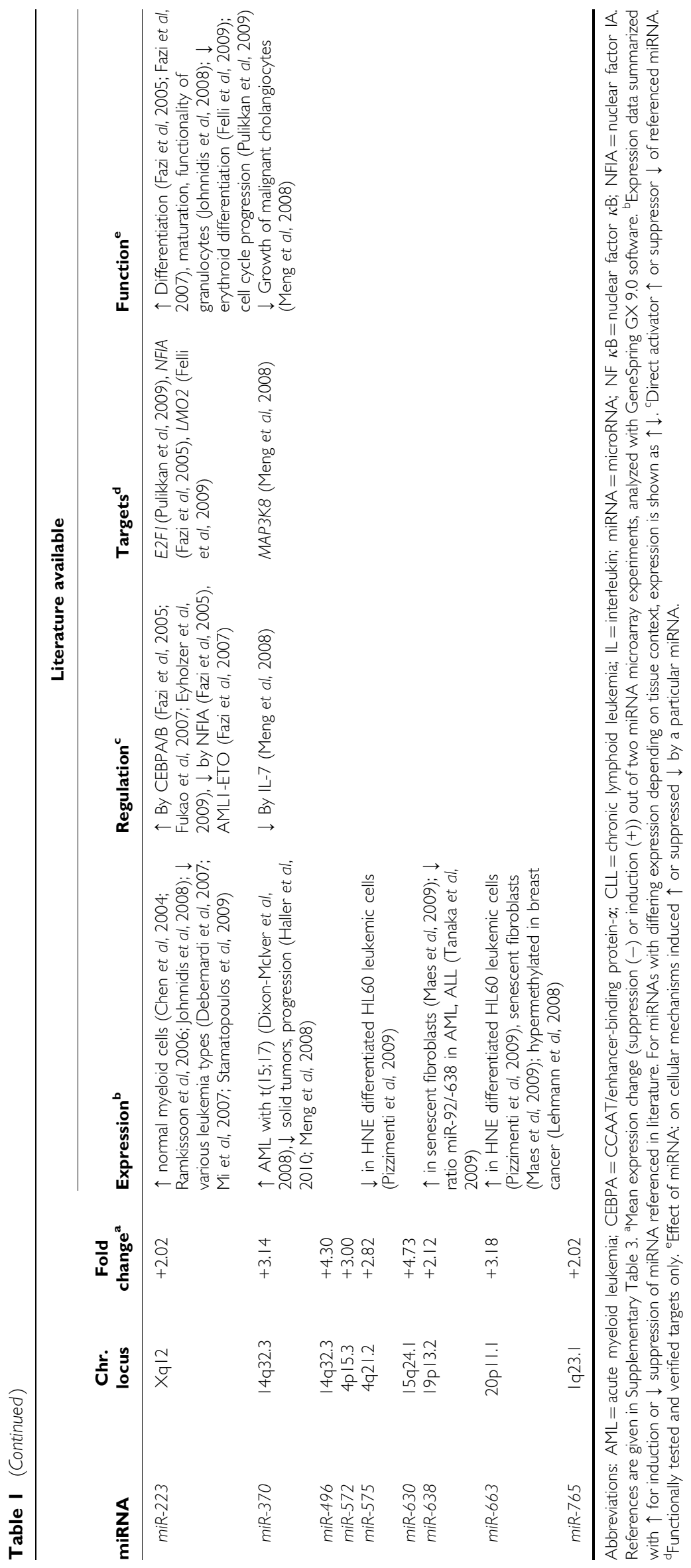



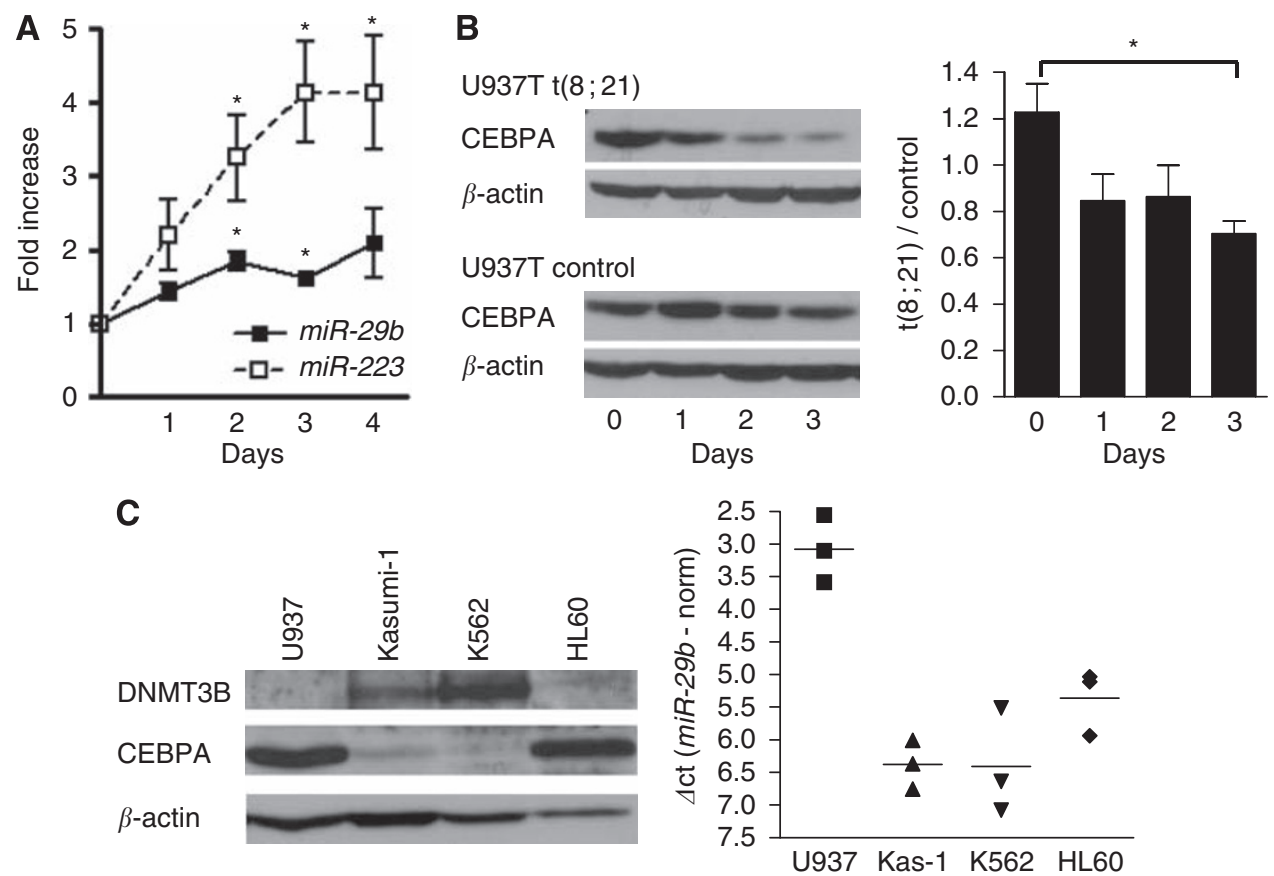

Figure I CEBPA mediates miR-29b expression in leukaemic cells. (A) Mature miR-29b expression was induced two-fold after restoring CEBPA function in leukemic Kasumi-I cells. The CEBPA target miR-223 was used as positive control for restored CEBPA function. Results are given as fold expression compared with untreated cells (day $0, * P<0.05$ ) and represent three independant experiments. (B) Conditional expression of AMLI-ETO abolished CEBPA protein expression in U937 leukemic cells (B, left) and suppressed miR-29b expression ( $* P<0.05$; $\mathbf{B}$, right). miR-29b expression is given as fold changes compared with the control cells U937-T ( $\Delta \Delta C_{\mathrm{t}}$-method) and represent three independant experiments. (C) Protein levels of the putative miR-29b activator CEBPA and the miR-29b target DNMT3B inversely correlated in leukemic cell lines (C, left). miR-29b expression correlated to CEBPA, and inversely correlated to DNMT3B protein levels (C) right). miR-29b expression is given as $\Delta C_{\mathrm{t}}$-values $\left(C_{\mathrm{t}}\left(\right.\right.$ miR-29b) $-C_{\mathrm{t}}$ (normalisation)) representing three independant experiments.

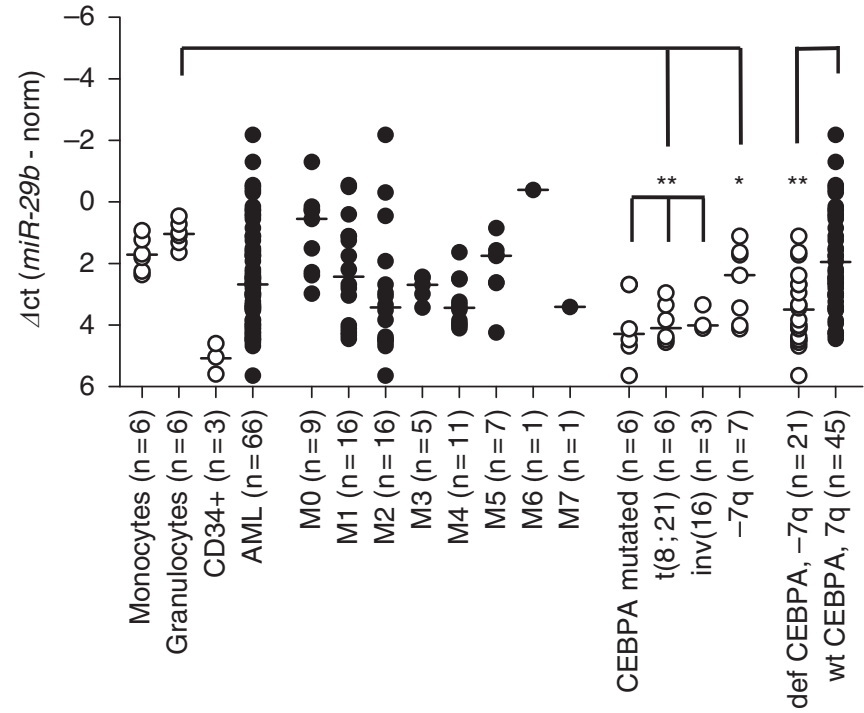

Figure 2 miR-29b expression in AML patients and healthy controls. miR-29b expression was assessed in samples from $66 \mathrm{AML}$ patients, in three samples of enriched CD34 + myeloid stem cells as well as in mature granulocytes and monocytes from six healthy volunteers. The cohort of consecutive AML samples showed suppressed miR-29b expression compared with granulocytes $(P=0.043)$. Patients with CEBPA deficiency (CEBPA mutations, $\mathrm{t}(8 ; 2 \mathrm{I})$, inv $(16)$ ) or chromosome $7 \mathrm{q}$ alterations (monosomy 7 or del(7q)) represented roughly one-third of the entire cohort and showed differences in miR-29b expression compared with granulocytes from healthy volunteers (*** $P<0.00$ I for all three subgroups with deficient CEBPA function, and $* P<0.05$ for $-7 q)$ as well as compared with the remaining $45 \mathrm{AML}$ patients (wt CEBPA and 7q, $* * P<0.001$ ). miR-29b expression was not suppressed in the remaining 45 patients (wt CEBPA and $7 q$ ) if compared with mature granulocytes $(P=0.182, N S)$. Expression levels are given as $\Delta C_{\mathrm{t}}$-values $\left(C_{\mathrm{t}}\left(\right.\right.$ miR-29b) $-C_{\mathrm{t}}($ normalisation $\left.)\right)$.
A computational analysis of the conserved region spanning -682 bp upstream to +296 bp downstream of the pri-miR-29a/bl transcription start site indicated six potential CEBP binding sites (Figure 4A). Using luciferase reporter assays, we observed that CEBPA, indeed, activated the entire conserved promoter region two-fold in a dose-dependent manner (Figure 4A). Deletion and mutation constructs of the pri-miR-29a/b1 promoter identified a CEBP binding site located +15 to +29 bp immediately downstream of the transcription start site to be responsible for CEBPAmediated activation of the pri-miR-29a/b1 promoter (Figure 4B). Chromatin immunoprecipitation assays in myeloid leukaemic cells confirmed that CEBPA is, in fact, binding in vivo to this part of the pri-miR-29a/b1 locus as suggested by the luciferase experiments (Figure 4C): both endogenous CEBPA in U937 cells and exogenous CEBPA in Kasumi-CEBPA-ER were binding to the CEBPA site located +15 to $+29 \mathrm{bp}$ downstream of the pri-miR-29a/b1 transcript start.

Furthermore, we sought to exclude additional functional CEBPA-binding sites in the non-conserved region directly upstream of the pre-miR29a and -29b1 stem structures. Such additional CEBPA responsive promoter elements were reported for CEBPA regulation of miR-223 (Fazi et al, 2005; Eyholzer et al, 2009) or miR-661 (Reddy et al, 2009). Although the computational sequence analysis of $2.2 \mathrm{~kb}$ upstream of the pre-miR-29b1 stem indicated four putative CEBP binding sites, we found that none of them was CEBPA responsive in luciferase assays (data not shown). We thus conclude that CEBPA activates $m i R-29 a / b$ expression through direct binding to a single site in the conserved promoter region of the pri-miR-29a/b1 transcript on chromosome $7 \mathrm{q} 32.3$.

\section{DISCUSSION}

The transcription factor CEBPA is a master regulator within normal haematopoiesis (Pabst and Mueller, 2007; Koschmieder 
A

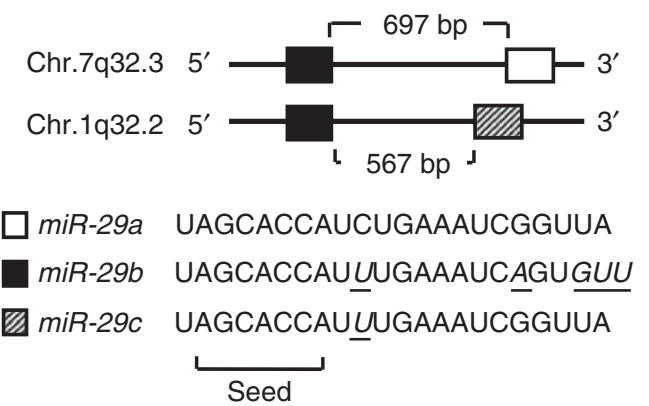

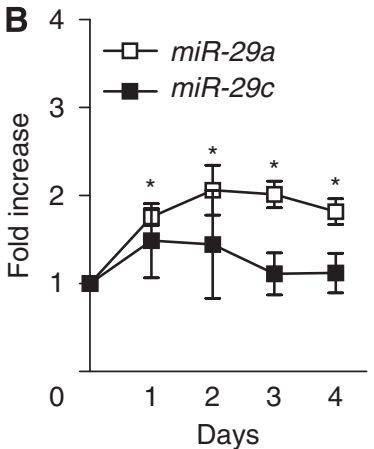
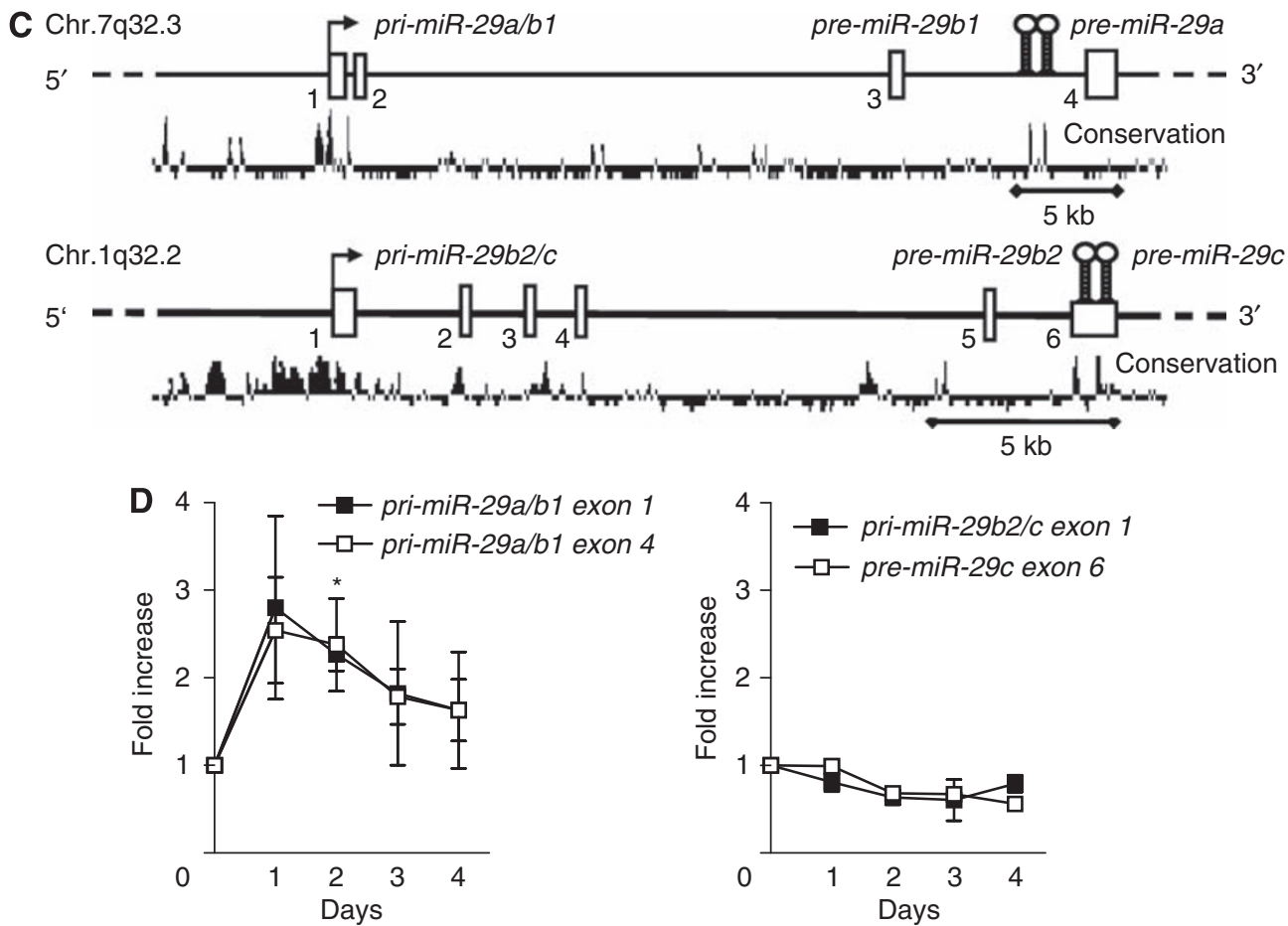

Figure 3 CEBPA activates miR-29b at the pri-miR-29a/b/ locus on chromosome 7q32.3. (A) miR-29b belongs to a family of three members (a, b, $c$ ), encoded on two loci on chromosome Iq32.2 and chromosome 7q32.3. Although miR-29b is encoded on both chromosomes, the mature miR-29b sequence encoded from the two loci is identical. (B) In addition to miR-29b, only mature miR-29a is induced after CEBPA restoration, but not mature miR-29c. (C) miR-29 family members are generated from two polymerase II primary transcripts: pri-miR-29a/b/ (GenBank accession number EU I54353) and pri-miR-29b2/c (EUI5435I and EUI54352). These primary transcripts are processed to double stranded precursors (pre-miR-29) and ultimately to the mature single stranded miR-29. Both pri-miR-29s are highly conserved in their putative promoter region and in the pre-miR-29 stem sequences, encoded in the last intron (pre-miR-29a, -b/) on chr.7q32.3 and the last exon (pre-miR-29b2, -c) on chr. I 32.2 respectively. (D) Primer pairs dispersed over the pri-miR$29 a / b /$ and pri-miR-29b2/c confirmed induction of the pri-miR-29a/b/ locus on chr.7q32.3 (D, left), whereas the pri-miR-29b2/c on chromosome I.q32.2 is not affected by CEBPA expression (D, right). Results are given as fold expression compared with untreated cells (day $0, * P<0.05)$ and represent three independant experiments.

et al, 2009). Increasing evidence indicates that CEBPA is exerting its regulatory effects, at least in part, by direct regulation of specific miRNAs. Fazi et al (2005) first identified $m i R-223$ as a direct target of CEBPA. The activation of $m i R-223$ by CEBPA triggers granulocytic differentiation and maturation (Fazi et al, 2005, 2007). Recently, miR-661 was reported to be another direct CEBPA target miRNA. miR-661 suppresses the metastatic tumour antigen 1 , a gene broadly upregulated in human cancer (Reddy et al, 2009).

In this study, we sought to identify the pattern of miRNAs that are regulated by CEBPA in haematopoietic cells. Using leukaemic Kasumi-1 cells with conditionally inducible CEBPA function (Pabst et al, 2001a), we determined the expression changes of 470 human miRNAs. We identified 18 miRNAs, whose expression levels were changed more than two-fold after restoring CEBPA function: $m i R-98, m i R-181 b, m i R-197, m i R-210, m i R-342, m i R-432$, $m i R-550$, and $m i R-776$ were suppressed, whereas $m i R-29 b$, $m i R-223, \quad m i R-370, \quad m i R-496, \quad m i R-572, \quad m i R-575, \quad m i R-630$, $m i R-638, m i R-663$, and $m i R-765$ were induced compared with their expression levels before CEBPA induction. As expected, the previously identified CEBPA target $m i R-223$ was induced more than two-fold. In contrast, $m i R-661$ levels remained stable in our system of myeloid leukaemic cells. As miR-661 activation by CEBPA was reported to be involved in some solid tumours (Reddy et al, 2009), it may be less important in haematopoietic cells.

So far, two studies described miRNA expression patterns associated with AML patients with CEBPA mutations (JongenLavrencic et al, 2008; Marcucci et al, 2008). In accordance with our observations, $m i R-181$ family members were induced in patients with CEBPA mutations in both studies. Remarkably, however, these authors have not reported other miRNAs detected by our 
A

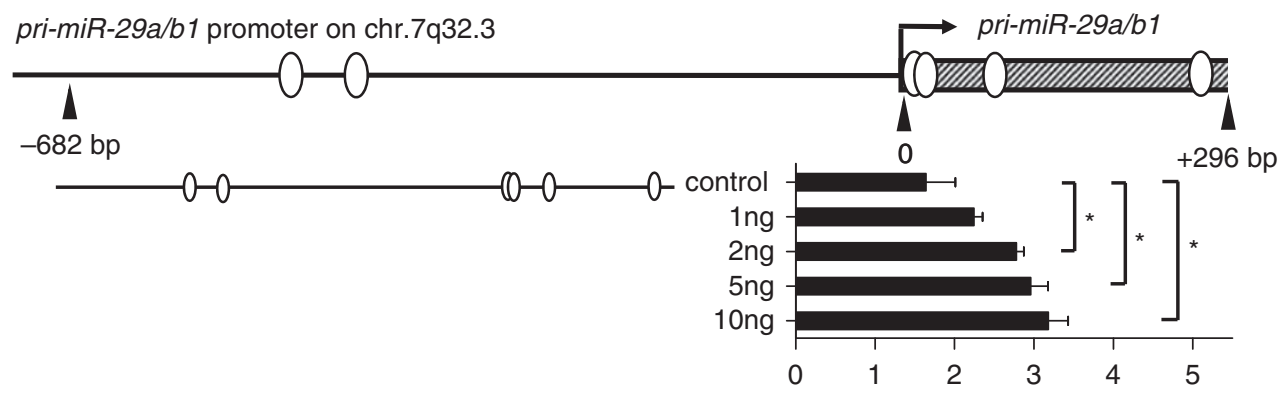

B
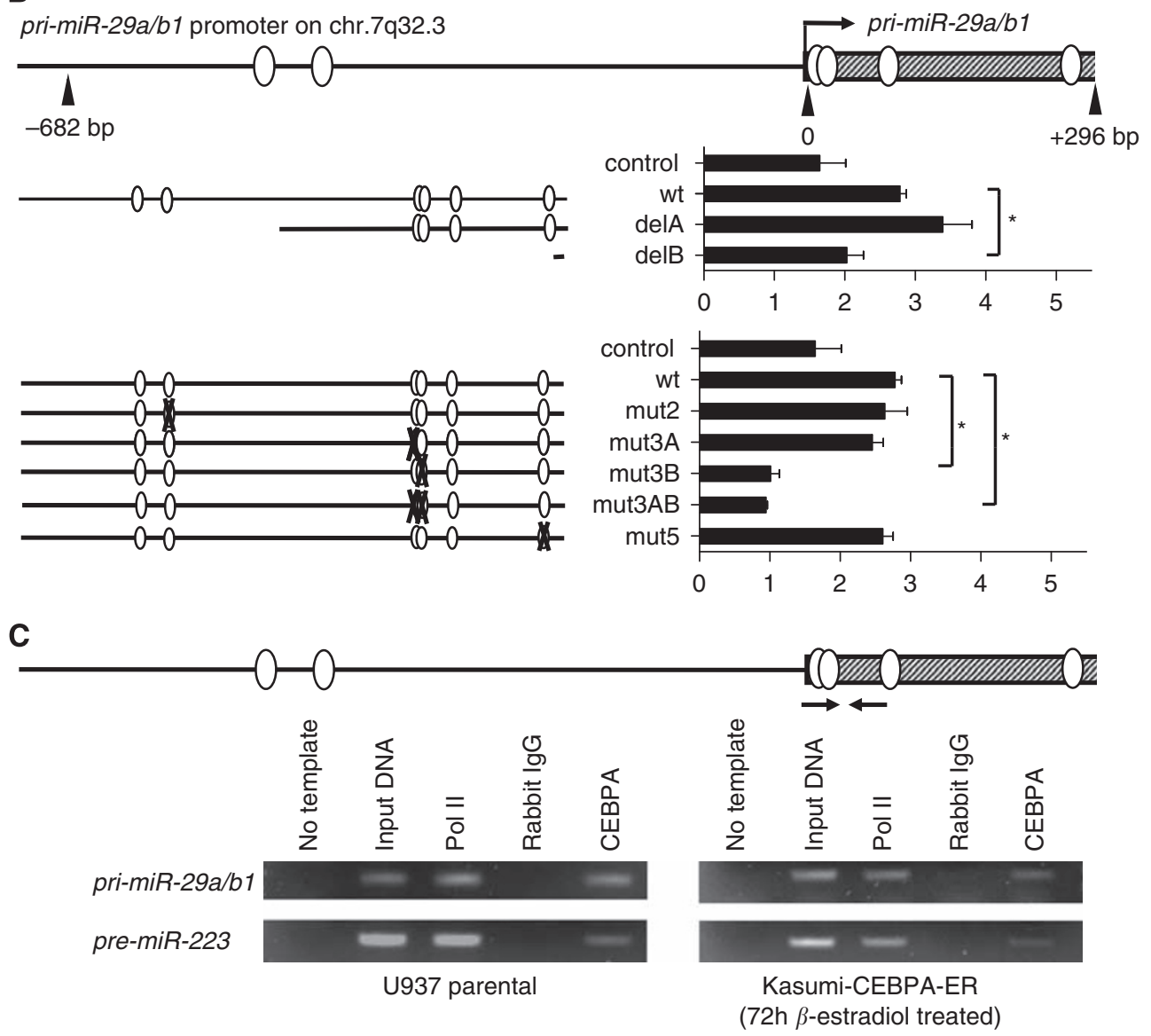

Figure 4 CEBPA activates the pri-miR29a/b/ promoter on chromosome 7q32.3. Results represent three independant experiments and are given as fold changes compared with the empty pcDNA3 expression vector; $* P<0.05$, control: empty pGL3b luciferase vector. (A) A computational analysis of the highly conserved region spanning -682 to +296 bp across the pri-miR-29a/b/ transcription start site resulted in six putative CEBPA binding sites. This sequence was cloned into the pGL3b luciferase vector and $200 \mathrm{ng}$ of promoter construct were transfected with I-I0 ng of CEBPA expression plasmid. (B) The CEBPA binding site +15 to +29 bp downstream of the pri-miR-29a/b/ transcription start was identified to be responsible for pri-miR-29alb 1 activation. Deleted (B, upper panel) or mutated (B, lower panel) pri-miR-29a/b / promoter construct (200 ng) were transfected with 2 ng CEBPA expression plasmid. (C) Chromatin immunoprecipitation (ChIP) assays confirmed binding of CEBPA to the site in the pri-miR-29a/b/ transcription start region. Endogenous CEBPA of U937 cells (C, left) and exogenous CEBPA in Kasumi-I-CEBPA-ER cells (C, right) were binding to the CEBPA site identified by the luciferase assays above. Binding of CEBPA to the regulatory element of pre-miR-223 was used as positive control for these ChIP experiments.

array approach. Possible explanations might be differences in the type of arrays used or differences arising from a comparison of results obtained from CEBPA restoration in a leukaemic cell line (as in this report) $v s$ differing miRNA patterns seen in patients with or without $C E B P A$ mutations.

Among the 18 identified miRNAs in our approach, we decided to dissect the molecular mechanisms involved in CEBPAdependent regulation of $m i R-29 b$ based on its increasingly recognised importance for normal haematopoiesis and leukaemo- gensis. Suppressed $m i R-29$ levels have been shown to be associated with disease progression in chronic lymphoid leukemia patients (Calin et al, 2005; Stamatopoulos et al, 2009; Visone et al, 2009). In AML, $m i R-29$ suppression is associated to translocations involving the $M L L$ oncogene ( $\mathrm{Li}$ et al, 2008), but it is induced in patients with NPM1 mutations in the absence of FLT3-ITD alterations (Garzon et al, 2008). Consistent with these expression data suggesting tumour-suppressive properties, $m i R-29$ is reported to trigger differentiation (Wang et al, 2008; Garzon et al, 2009b; 
Li et al, 2009) and apoptosis (Mott et al, 2007; Garzon et al, 2009a; Park et al, 2009; Xiong et al, 2009) in various tissues as well as having anti-invasive and anti-proliferative properties in solid tumours (Muniyappa et al, 2009; Xiong et al, 2009).

Several potential oncogenes have been reported to be silenced by miR-29, such as Tcl1 (Pekarsky et al, 2006), YY1 (Wang et al, 2008), CXXC6, and CDK6 (Garzon et al, 2009a), the p53 upstream inhibitors $p 85 \alpha$ and $C D C 42$ (Park et al, 2009), and the antiapoptotic Bcl2 family members $\mathrm{Bcl} 2$ and $\mathrm{Mcl} 1$ (Mott et al, 2007; Garzon et al, 2009a; Xiong et al, 2009). Importantly, miR-29 family members were also reported to have an important role in preventing epigenetic silencing of tumour suppressors due to de novo methylation in cancer, as they directly suppress DNMT3A and B (Fabbri et al, 2007; Garzon et al, 2009b).

Despite the variety of reported $m i R-29$ downstream effects, little is known so far on how $m i R-29$ expression is regulated itself. Chang et al (2008) first described the conserved promoter regions of both miR-29 family clusters on chromosome 1q32.2 ( $m i R-29 b 2 /$ c) and chromosome 7q32.3 (miR-29a/b1). They showed that both clusters were suppressed by the oncogenic transcription factor Myc in B-cell lymphoma. Wang et al (2008) proposed that the $m i R$ $29 \mathrm{~b} / \mathrm{c}$ cluster on chromosome 1 is suppressed in rhabdomyosarcoma through $\mathrm{NF \kappa B} / \mathrm{YY} 1$ via the same conserved upstream promoter region.

In this study, we report that the haematopoietic master transcription factor CEBPA is inducing $m i R-29 b$ expression. We observed that $m i R-29 b$ is suppressed in AML patients with disrupted CEBPA function. This comprises AML patients with CEBPA mutations or with suppressed CEBPA function because of $t(8,21)$ or inv(16) chromosome aberrations (Pabst et al, 2001a,b; Helbling et al, 2005). We also confirmed recent observations by others (Garzon et al, 2009a) that miR-29b is suppressed in AML patients with alterations of chromosome 7 (monosomy 7 or $\operatorname{del}(7 \mathrm{q})$ ). Interestingly, functional analysis of CEBPA-mediated miR-29b expression indicated that only the $m i R-29 a / b 1$ locus on chromosome 7q32.3 is activated by CEBPA, whereas $m i R-29 b 2 / c$ expression is not affected by CEBPA. This was surprising as the conserved promoter region upstream of the $m i R-29 b 2 / c$ primary transcript on chromosome 1q32.2 (Chang et al, 2008) also indicated several putative CEBPA binding sites in a computational analysis. However, we found that none of them turned out to be functional. The finding that CEBPA induces miR-29b expression only from its chromosome $7 \mathrm{q} 32.3$ locus ( $m i R-29 a / b 1$ cluster) provides a rationale for $m i R-29 b$ suppression observed in patients with alterations of chromosome 7 .

In summary, using miRNA microarrays, we found that CEBPA affects the expression of a defined subset of 18 miRNAs in human AML cells. Among them, we identified the $m i R-29 a / b 1$ cluster encoded on chromosome $7 \mathrm{q} 32.3$ to be directly activated by CEBPA. The findings of our study suggest a rationale for $m i R-29 b$ suppression in AML patients with disrupted CEBPA function or with aberrations on chromosome 7.

\section{ACKNOWLEDGEMENTS}

We thank Caroline Hammer for support with the miRNA microarrays; Deborah Shan for help with preparing patient samples; Mathias Jenal for technical advice with the ChIP assays; Simon Häfliger, Sarah Mans, Andreas Steege and Duttu Vallabhapurapu for helpful comments; Elisabeth Ischii and Elke Beutler for help with CD34 + sample collection; and all patients and volunteers for donating samples. This work was supported by grants from the Swiss National Science Foundation SF 310000-109388 and 310030-127509 (to TP).

Contributions: ME designed and performed research and wrote the article; SS performed research; LW and BUM analysed data; and TP designed research and wrote the article.

\section{Conflict of interest}

The authors declare no conflicts of interest.

Supplementary Information accompanies the paper on British Journal of Cancer website (http://www.nature.com/bjc)

\section{REFERENCES}

Ambros V, Chen X (2007) The regulation of genes and genomes by small RNAs. Development 134: 1635-1641

Calin GA, Ferracin M, Cimmino A, Di Leva G, Shimizu M, Wojcik SE, Iorio MV, Visone R, Sever NI, Fabbri M, Iuliano R, Palumbo T, Pichiorri F, Roldo C, Garzon R, Sevignani C, Rassenti L, Alder H, Volinia S, Liu CG, Kipps TJ, Negrini M, Croce CM (2005) A microRNA signature associated with prognosis and progression in chronic lymphocytic leukemia. N Engl J Med 353: 1793-1801

Chang TC, Yu D, Lee YS, Wentzel EA, Arking DE, West KM, Dang CV, Thomas-Tikhonenko A, Mendell JT (2008) Widespread microRNA repression by Myc contributes to tumorigenesis. Nat Genet 40: 43-50

Eyholzer M, Schmid S, Schardt JA, Haefliger S, Mueller BU, Pabst T (2009) Complexity of miR-223 regulation by CEBPA in human AML. Leuk Res 34(5): $672-676$

Fabbri M, Garzon R, Andreeff M, Kantarjian HM, Garcia-Manero G, Calin GA (2008) MicroRNAs and noncoding RNAs in hematological malignancies: molecular, clinical and therapeutic implications. Leukemia 22: $1095-1105$

Fabbri M, Garzon R, Cimmino A, Liu Z, Zanesi N, Callegari E, Liu S, Alder H, Costinean S, Fernandez-Cymering C, Volinia S, Guler G, Morrison CD, Chan KK, Marcucci G, Calin GA, Huebner K, Croce CM (2007) MicroRNA29 family reverts aberrant methylation in lung cancer by targeting DNA methyltransferases 3A and 3B. Proc Natl Acad Sci USA 104: 15805-15810

Fazi F, Racanicchi S, Zardo G, Starnes LM, Mancini M, Travaglini L, Diverio D, Ammatuna E, Cimino G, Lo-Coco F, Grignani F, Nervi C (2007) Epigenetic silencing of the myelopoiesis regulator microRNA-223 by the AML1/ETO oncoprotein. Cancer Cell 12: 457-466
Fazi F, Rosa A, Fatica A, Gelmetti V, De Marchis ML, Nervi C, Bozzoni I (2005) A minicircuitry comprised of microRNA-223 and transcription factors NFI-A and C/EBPalpha regulates human granulopoiesis. Cell 123: $819-831$

Fukao T, Fukuda Y, Kiga K, Sharif J, Hino K, Enomoto Y, Kawamura A, Nakamura K, Takeuchi T, Tanabe M (2007) An evolutionarily conserved mechanism for microRNA-223 expression revealed by microRNA gene profiling. Cell 129: 617-631

Garzon R, Croce CM (2008) MicroRNAs in normal and malignant hematopoiesis. Curr Opin Hematol 15: $352-358$

Garzon R, Garofalo M, Martelli MP, Briesewitz R, Wang L, FernandezCymering C, Volinia S, Liu CG, Schnittger S, Haferlach T, Liso A, Diverio D, Mancini M, Meloni G, Foa R, Martelli MF, Mecucci C, Croce CM, Falini B (2008) Distinctive microRNA signature of acute myeloid leukemia bearing cytoplasmic mutated nucleophosmin. Proc Natl Acad Sci USA 105: 3945-3950

Garzon R, Heaphy CE, Havelange V, Fabbri M, Volinia S, Tsao T, Zanesi N, Kornblau SM, Marcucci G, Calin GA, Andreeff M, Croce CM (2009a) MicroRNA 29b functions in acute myeloid leukemia. Blood 114(26): $5331-5341$

Garzon R, Liu S, Fabbri M, Liu Z, Heaphy CE, Callegari E, Schwind S, Pang J, Yu J, Muthusamy N, Havelange V, Volinia S, Blum W, Rush LJ, Perrotti D, Andreeff M, Bloomfield CD, Byrd JC, Chan K, Wu LC, Croce CM, Marcucci G (2009b) MicroRNA-29b induces global DNA hypomethylation and tumor suppressor gene reexpression in acute myeloid leukemia by targeting directly DNMT3A and 3B and indirectly DNMT1. Blood 113: 6411-6418 
Helbling D, Mueller BU, Timchenko NA, Hagemeijer A, Jotterand M, Meyer-Monard S, Lister A, Rowley JD, Huegli B, Fey MF, Pabst T (2004) The leukemic fusion gene AML1-MDS1-EVI1 suppresses CEBPA in acute myeloid leukemia by activation of calreticulin. Proc Natl Acad Sci USA 101: $13312-13317$

Helbling D, Mueller BU, Timchenko NA, Schardt J, Eyer M, Betts DR, Jotterand M, Meyer-Monard S, Fey MF, Pabst T (2005) CBFB-SMMHC is correlated with increased calreticulin expression and suppresses the granulocytic differentiation factor CEBPA in AML with inv(16). Blood 106: 1369-1375

Johnnidis JB, Harris MH, Wheeler RT, Stehling-Sun S, Lam MH, Kirak O, Brummelkamp TR, Fleming MD, Camargo FD (2008) Regulation of progenitor cell proliferation and granulocyte function by microRNA-223. Nature 451: $1125-1129$

Jongen-Lavrencic M, Sun SM, Dijkstra MK, Valk PJ, Lowenberg B (2008) MicroRNA expression profiling in relation to the genetic heterogeneity of acute myeloid leukemia. Blood 111: 5078-5085

Kluiver J, Kroesen BJ, Poppema S, van den Berg A (2006) The role of microRNAs in normal hematopoiesis and hematopoietic malignancies. Leukemia 20: 1931 - 1936

Koschmieder S, Halmos B, Levantini E, Tenen DG (2009) Dysregulation of the C/EBPalpha differentiation pathway in human cancer. J Clin Oncol 27: $619-628$

Lawrie CH (2007) MicroRNAs and haematology: small molecules, big function. Br J Haematol 137: 503-512

Li Z, Hassan MQ, Jafferji M, Aqeilan RI, Garzon R, Croce CM, van Wijnen AJ, Stein JL, Stein GS, Lian JB (2009) Biological functions of miR-29b contribute to positive regulation of osteoblast differentiation. J Biol Chem 284: $15676-15684$

Li Z, Lu J, Sun M, Mi S, Zhang H, Luo RT, Chen P, Wang Y, Yan M, Qian Z, Neilly MB, Jin J, Zhang Y, Bohlander SK, Zhang DE, Larson RA, Le Beau MM, Thirman MJ, Golub TR, Rowley JD, Chen J (2008) Distinct microRNA expression profiles in acute myeloid leukemia with common translocations. Proc Natl Acad Sci USA 105: 15535-15540

Lin RK, Hsu HS, Chang JW, Chen CY, Chen JT, Wang YC (2007) Alteration of DNA methyltransferases contributes to $5^{\prime} \mathrm{CpG}$ methylation and poor prognosis in lung cancer. Lung Cancer 55: 205-213

Marcucci G, Maharry K, Radmacher MD, Mrozek K, Vukosavljevic T, Paschka P, Whitman SP, Langer C, Baldus CD, Liu CG, Ruppert AS, Powell BL, Carroll AJ, Caligiuri MA, Kolitz JE, Larson RA, Bloomfield CD (2008) Prognostic significance of, and gene and microRNA expression signatures associated with, CEBPA mutations in cytogenetically normal acute myeloid leukemia with high-risk molecular features: a Cancer and Leukemia Group B Study. J Clin Oncol 26: 5078-5087

Mott JL, Kobayashi S, Bronk SF, Gores GJ (2007) mir-29 regulates Mcl-1 protein expression and apoptosis. Oncogene 26: 6133-6140

Mueller BU, Pabst T (2006) C/EBPalpha and the pathophysiology of acute myeloid leukemia. Curr Opin Hematol 13: 7-14

Muniyappa MK, Dowling P, Henry M, Meleady P, Doolan P, Gammell P, Clynes M, Barron N (2009) MiRNA-29a regulates the expression of numerous proteins and reduces the invasiveness and proliferation of human carcinoma cell lines. Eur J Cancer 45: 3104-3118

Pabst T, Mueller BU (2007) Transcriptional dysregulation during myeloid transformation in AML. Oncogene 26: 6829-6837

Pabst T, Mueller BU, Harakawa N, Schoch C, Haferlach T, Behre G, Hiddemann W, Zhang DE, Tenen DG (2001a) AML1-ETO downregulates the granulocytic differentiation factor C/EBPalpha in $\mathrm{t}(8 ; 21)$ myeloid leukemia. Nat Med 7: 444-451
Pabst T, Mueller BU, Zhang P, Radomska HS, Narravula S, Schnittger S, Behre G, Hiddemann W, Tenen DG (2001b) Dominant-negative mutations of CEBPA, encoding CCAAT/enhancer binding protein-alpha (C/EBPalpha), in acute myeloid leukemia. Nat Genet 27: 263-270

Park SY, Lee JH, Ha M, Nam JW, Kim VN (2009) miR-29 miRNAs activate p53 by targeting p85 alpha and CDC42. Nat Struct Mol Biol 16: 23-29

Pekarsky Y, Santanam U, Cimmino A, Palamarchuk A, Efanov A, Maximov V, Volinia S, Alder H, Liu CG, Rassenti L, Calin GA, Hagan JP, Kipps T, Croce CM (2006) Tcl1 expression in chronic lymphocytic leukemia is regulated by miR-29 and miR-181. Cancer Res 66: 11590-11593

Pelosi E, Labbaye C, Testa U (2009) MicroRNAs in normal and malignant myelopoiesis. Leuk Res 33: 1584-1593

Peltier HJ, Latham GJ (2008) Normalization of microRNA expression levels in quantitative RT-PCR assays: identification of suitable reference RNA targets in normal and cancerous human solid tissues. RNA 14: $844-852$

Radomska HS, Huettner CS, Zhang P, Cheng T, Scadden DT, Tenen DG (1998) CCAAT/enhancer binding protein alpha is a regulatory switch sufficient for induction of granulocytic development from bipotential myeloid progenitors. Mol Cell Biol 18: $4301-4314$

Reddy SD, Pakala SB, Ohshiro K, Rayala SK, Kumar R (2009) MicroRNA661, a c/EBPalpha target, inhibits metastatic tumor antigen 1 and regulates its functions. Cancer Res 69: $5639-5642$

Rhee I, Bachman KE, Park BH, Jair KW, Yen RW, Schuebel KE, Cui H, Feinberg AP, Lengauer C, Kinzler KW, Baylin SB, Vogelstein B (2002) DNMT1 and DNMT3b cooperate to silence genes in human cancer cells. Nature 416: $552-556$

Robertson KD, Uzvolgyi E, Liang G, Talmadge C, Sumegi J, Gonzales FA, Jones PA (1999) The human DNA methyltransferases (DNMTs) 1, 3a and $3 \mathrm{~b}$ : coordinate mRNA expression in normal tissues and overexpression in tumors. Nucleic Acids Res 27: 2291-2298

Rosenbauer F, Tenen DG (2007) Transcription factors in myeloid development: balancing differentiation with transformation. Nat Rev Immunol 7: 105-117

Shivdasani RA (2006) MicroRNAs: regulators of gene expression and cell differentiation. Blood 108: 3646-3653

Stamatopoulos B, Meuleman N, Haibe-Kains B, Saussoy P, Van Den Neste E, Michaux L, Heimann P, Martiat P, Bron D, Lagneaux L (2009) microRNA-29c and microRNA-223 down-regulation has in vivo significance in chronic lymphocytic leukemia and improves disease risk stratification. Blood 113: $5237-5245$

Tenen DG (2003) Disruption of differentiation in human cancer: AML shows the way. Nat Rev Cancer 3: 89-101

Visone R, Rassenti LZ, Veronese A, Taccioli C, Costinean S, Aguda BD, Volinia S, Ferracin M, Palatini J, Balatti V, Alder H, Negrini M, Kipps TJ, Croce CM (2009) Karyotype-specific microRNA signature in chronic lymphocytic leukemia. Blood 114: 3872-3879

Wang H, Garzon R, Sun H, Ladner KJ, Singh R, Dahlman J, Cheng A, Hall BM, Qualman SJ, Chandler DS, Croce CM, Guttridge DC (2008) NF-kappaB-YY1-miR-29 regulatory circuitry in skeletal myogenesis and rhabdomyosarcoma. Cancer Cell 14: 369-381

Xiong Y, Fang JH, Yun JP, Yang J, Zhang Y, Jia WH, Zhuang SM (2009) Effects of MicroRNA-29 on apoptosis, tumorigenicity, and prognosis of hepatocellular carcinoma. Hepatology 51(3): 836-845

Zhang DE, Zhang P, Wang ND, Hetherington CJ, Darlington GJ, Tenen DG (1997) Absence of granulocyte colony-stimulating factor signaling and neutrophil development in CCAAT enhancer binding protein alpha-deficient mice. Proc Natl Acad Sci USA 94: 569-574 